\title{
Submitted from BMJ Quality \\ Improving oxygen prescribing rates by tailoring interventions for specific healthcare professional groups
}

\author{
Sebastian Helliar
}

To cite: Helliar S. Improving oxygen prescribing rates by tailoring interventions for specific healthcare professional groups. BMJ Quality Improvement Reports 2016;5:u209520.w4033. doi:10.1136/bmjquality. u209520.w4033

Received 28 July 2016 Revised 24 October 2016

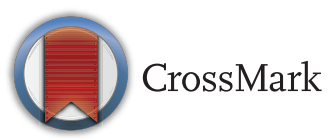

NHS, United Kingdom

Correspondence to Sebastian Helliar sebhelliar@ gmail.com

\section{ABSTRACT}

Oxygen prescription remains a nationwide problem. The dangers associated with unregulated oxygen administration are well described in the literature with the potential for serious harm in patients with chronic hypercapnia, as well as potentially delaying discharge in patients who are administered it without a prescription. This project identifies poor compliance with regional and national standards and sets out to improve the frequency of oxygen prescribing on a cardiology ward. By studying the problem at a Somerset district general hospital we identified two main groups of professionals responsible for the poor compliance, nursing staff (who administer the oxygen) and junior doctors (who should prescribe it). A series of interventions was designed to firstly raise awareness of the problem within these two groups before going on to target each group with a further intervention over 24 weeks. At baseline we found only $11.3 \%$ of patients receiving oxygen had it prescribed. At the end of the project this had improved to $69.6 \%$. We also found that following raised awareness in the nursing staff and introduction of a bedside warning the number of patients receiving oxygen on the ward fell by $35 \%$. In conclusion, this project outlines a strategy for improving oxygen prescribing rates on a medical ward. By targeting different populations we had hoped to see a cumulative improvement after each improvement cycle, however, some resistance from junior doctors in engaging with our third intervention was reflected with a slight decrease in prescribing rates. Further work should address this issue and look to apply this strategy across a wider clinical area with a greater sample size to see if the results are replicable on a larger scale.

\section{PROBLEM}

Despite the clear BTS guidelines safe and consistent oxygen prescribing remains a nationwide problem. The potential for harm from over oxygenation is well documented, particularly in patients at risk of carbon dioxide retention, and type 2 respiratory failure. $^{12}$

Whilst working on a 27 bed cardiology ward in a large Somerset district general hospital the project team encountered this problem first hand.

The two main problems identified are that oxygen is not regularly being prescribed and that Oxygen is commonly being administered without a valid prescription and without documented target saturation.

Our SMART aim was to improve rates of oxygen prescription on the cardiology ward for patients receiving oxygen to $75 \%$ by August 2015.

This project proposes a series of interventions to increase compliance with both national and trust guidelines. These guidelines recommend that all oxygen should be prescribed, unless it is administered in the emergency situation, in which case it should be promptly reviewed.

I worked under the supervision of my clinical supervisor and liaised with the hospital's respiratory consultant body and Oxygen audit lead. ${ }^{1}$

\section{BACKGROUND}

Oxygen is a widely used drug in the NHS with the national BTS audit reporting just over $14 \%$ of inpatients sampled received oxygen. An oxygen tap can be found at most bedsides and the administration of oxygen is very simple, this contributes to it often being started without medical review or without a valid prescription. It is a very powerful drug which is effective in treating hypoxia, the causes of which are numerous. It is worth noting that oxygen is often incorrectly used in breathless patients, in studies into the use of oxygen in breathlessness without hypoxia researchers found that there was no significant improvement over placebo. ${ }^{1}$

Before oxygen is administered or prescribed an assessment of the patient should be done to identify any risk factors for carbon dioxide retention. In patients who chronically retain carbon dioxide $(\mathrm{C} 02)$ a degree of hypoxia is essential to maintain 
central respiratory drive. Removing this respiratory stimulus can lead to respiratory failure. One 2010 study showed that even short bursts of oxygen therapy, in the ambulance prior to admission, significantly increases mortality in this oxygen sensitive group. Inpatients with risk factors for hypercapnia are common, any of the following conditions can lead to retention; obesity, chronic obstructive pulmonary disease, chronic respiratory disease such as bronchiectasis, chest wall abnormalities and disorders of the neuromuscular junction. ${ }^{34}$

Following its prescription (which should include a target saturation) oxygen should be administered by nursing staff, who will choose the delivery device and oxygen flow rate. Changing devices and flow rates allows the target saturation to be reached and ongoing oxygen administration titrated to maintain the target. Oxygen should not be given without a valid prescription, the only exception to this is the emergency administration where intervention is required rapidly. BTS guidance states that even in the emergency situation the oxygen delivery and requirement should be promptly reviewed and prescribed appropriately.

Other projects addressing this issue have found difficulty changing the culture around oxygen prescribing. Good results have been shown when using visual reminders and one project in Bristol suggested this should be trialed in different centres, they found however difficulty sustaining the initial improvement seen after each plan, do, study, act cycle. ${ }^{5}$

Interestingly the NICE guidance for COPD, whilst providing some broad recommendations, states that local guidelines should be followed regarding oxygen therapy during acute exacerbations of the disease. ${ }^{6}$

\section{BASELINE MEASUREMENT}

Data was collected on the twenty-seven bed cardiology ward over a two month period to obtain a baseline measurement.

Data collection occurred daily, most frequently on the ward rounds. Patients receiving oxygen were identified and the medical administration charts were crosschecked for a valid prescription with a documented target oxygen saturation. For the baseline data collection the medical notes were reviewed to identify whether or not the patient had risk factors for hypercapnic respiratory failure, which 11 of the 53 did.

We then implemented our first improvement cycle with repeat data being collected prior to each further cycle commencing at approximately 6 week intervals.

At baseline, of the 53 patients who received oxygen only $11.3 \%(6 / 53)$ had a valid oxygen prescription. In patients with risk factors for hypercapnia $27.27 \%$ (3/11) had a valid prescription whilst $7.4 \%(3 / 42)$ of the remaining patients met the prescription standard.

The outcome measure of the project is the percentage of all patients who are receiving oxygen therapy that have a valid oxygen prescription (See supplementary file - "Point-of-care reminders, Cycle 2").

\section{DESIGN}

Throughout the project period we introduced 3 structured interventions. The first intervention was to convey the poor baseline data to the key groups of staff who could influence improvement in this problem. The second and third then each targeted one of these groups in an attempt to achieve a maintained improvement.

From very early on in the project we involved ward sister Jacqueline Phillips. She was instrumental in communicating and reinforcing our message to the large body of frequently rotating nursing staff. We communicated with the nursing staff through Sister Phillips as well as meeting informally with nursing staff members on the ward to promote engagement. The junior doctor team on the ward was much smaller and catalysing participation through the supervising consultant was simpler.

This project was led by Dr Sebastian Helliar, with supervision and assistance from consultant Dr Dan Mckenzie and ward sister Jacqueline Phillips.

\section{STRATEGY}

Improvement cycle 1 - Our first intervention aimed to raise awareness of the baseline data and the problems arising from poor compliance to the standard. We hypothesised that this alone might yield an improvement in prescribing rates. We planned to disseminate our baseline data to the relevant individuals. Following discussion with the ward sister the ward nursing staff and junior doctors were emailed a report of the baseline data. Pursuing a more personal approach this was followed up by meeting each individual staff member and discussing their role in the problem and what steps they could take for an improvement to occur. For the nursing staff this required an explanation of the potential dangers of oxygen therapy and an understanding that it should not be given without a valid prescription (except in the emergency setting). We also highlighted the importance of titrating delivery to target saturation. For the junior ward doctors the discussion centred around a change in oxygen prescribing practise, highlighting the need to assess new patients to the ward and consider prescribing oxygen proactively rather than reactively. This cycle ran from November 17th to December 22nd, when repeat data was collected showing an improvement. Prescribing rates rose to $30.8 \%$. We planned future cycles to improve compliance within these two groups of healthcare professionals.

Improvement cycle 2 - Our aim in cycle 2 was to prevent or reduce nurses giving oxygen to patients who did not have a prescription. Following review of previous improvement projects we hypothesised that a 
point-of-care reminder could achieve this. The second intervention was the introduction of a visual reminder at the point of oxygen administration (the oxygen gas taps). This targeted the nursing staff, a population we identified as being at least partly responsible for the poor compliance with the standard. These aids differed from previous projects' visual reminders as they do not attach to the oxygen taps themselves, they were placed on the wall above the taps. This allowed a much larger visual aid to be placed which we hoped would be less easily ignored. These highlighted the correct protocol for oxygen administration and reinforced the message delivered to the nursing staff in the first 'Improvement cycle', that oxygen should not be given without a valid prescription (unless in the emergency setting). A meeting was arranged with the respiratory consultants who approved the use of these 'reminders'. A copy of the reminder can be found in the 'supplementary materials' section. ${ }^{5}$ This cycle was delayed waiting for approval for the reminders and ran from 29th December to 23rd February. Data collected at the end of this cycle showed a greater improvement than before. Prescribing rates had risen to $76.5 \%$. We hoped continued improvement would be shown by focusing on junior doctors in the next cycle.

Improvement cycle 3 - The aim was to encourage doctors to consider patients oxygen requirements on admission to the ward and to prescribe in a more proactive manner, rather than the reactive manner in which oxygen is so often prescribed. We targeted junior doctors, the second group we identified as being responsible for safe oxygen prescription. We introduced a 'New patient clerking proforma' to the ward which was to be used whenever a patient was admitted, it served to provide a concise but thorough documentation of a patient's medical issues and history. On this proforma there was also a safety reminder for 'oxygen prescribed' with a tick box next to it. A 'Safety Checklist' was placed on the proforma prompting the admitting doctor to

\section{Improving oxygen prescribing rates by tailoring interventions for specific healthcare professional groups}

Run chart for project period November 2014 - May 2015

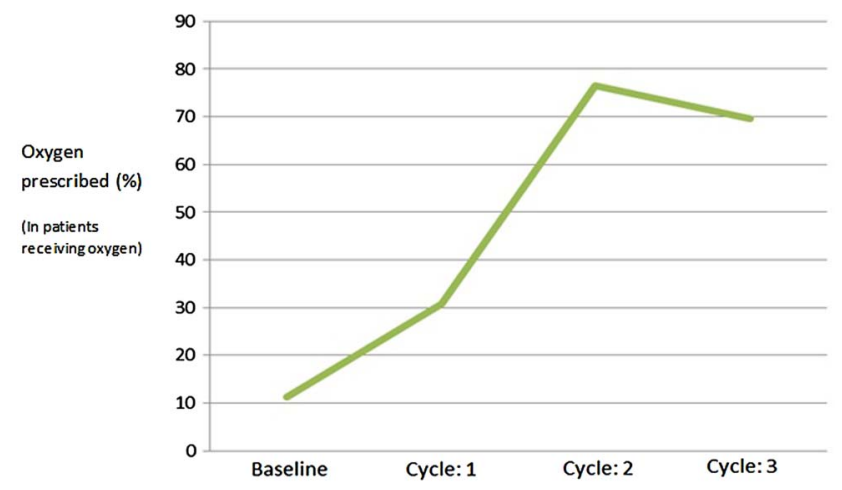

"Results run chart" prescribe oxygen as well as highlighting the need to review venous thromboembolism prophylaxis, antibiotics and treatment escalation plans. It also provides a lasting reminder which will be seen by future junior doctors rotating through the ward every 4 months. A copy of the proforma can be found in the 'supplementary materials' section. We hypothesised that this would yield further improvement however after the final data collection in early May prescribing rates had fallen to $69.5 \%$ (See supplementary file - "Admission proforma for completion by junior ward doctors, cycle 3").

\section{RESULTS}

Following improvement cycle one and dissemination of our baseline results the frequency of appropriate oxygen prescribing improved to $30.8 \%(8 / 26)$.

Following improvement cycle two and the introduction of visual reminders we found that the number of patients receiving oxygen in the 6 week period had dropped by $35 \%$ (17 vs 26 ) and of the patients receiving oxygen $76.5 \%(13 / 17)$ had a valid prescription.

Following improvement cycle three, the introduction of new patient proformas, we found that $69.6 \%(16 / 23)$ of patients receiving oxygen had a valid prescription.

Our baseline measurement showed $11.3 \%$ of patients receiving oxygen therapy had a valid prescription. Following the final data collection we found this had risen to $69.6 \%$.

\section{LESSONS AND LIMITATIONS}

Whilst the project has been a success it is worth noting several limitations that warrant consideration.

The size of this project limits the weight the results carry. Being conducted on one ward where a minority of patients received oxygen meant the sample sizes were small. Smaller samples such as these are far more susceptible to the influence of confounding factors, chance and bias. Future work should consider trials of the described strategies on larger populations and collect more data at more frequent time intervals to test if results are statistically significant. It would also be interesting to study the effects of these interventions in other centres giving an idea of their generalisability. Whilst we believe similar improvement would be seen, this has yet to be tested or proven.

In hindsight it would have been appropriate to start this project with an even smaller sample size. This would have allowed a trial of the proposed strategies on a smaller group, requiring less time, industry and resources to attain a result. Any strategies found to be successful on small groups could then be scaled up as described above in a more streamlined fashion.

Continuing the period of measurement on for a longer period following the final cycle would provide valuable information on the sustainability of the improvement seen. We saw a fall in prescribing between cycles 2 and 3 . It would be interesting to see if this 
merely represented a leveling out of the improvement or the start of a more significant decline.

The project was conducted on one ward with a small number of staff, a high proportion of them regular. This made it relatively easy to personally meet with them all and explain the projects findings and aims and encourage change, this almost certainly contributed to the significant improvement seen. If this project was to be implemented into a larger clinical area the effect may be diminished or lost as mass email communication is much less memorable.

Engagement of the groups we identified as our targets differed. We were fortunate enough to have the full backing of the ward sister who actively got involved in the project and encouraged the nursing staff to engage too. The visual reminders were actually produced and placed by the ward nurses and this can only have increased their impact. Conversely the junior doctors saw the intervention that targeted them (Cycle3) as extra paperwork and considerable resistance was met. This is likely to explain why Cycle2 was our most effective cycle whilst Cycle3 saw a small regression from $76.5 \%$ to $69.6 \%$.

\section{CONCLUSION}

The project has been successful in increasing oxygen prescribing rates from $11.3 \%$ to $69.5 \%$. This improvement is similar to those described by other groups in this area. Like previous QI work in Bristol we found the greatest improvement following introduction of point-of-care reminders and further projects should look to incorporate these into their strategy. ${ }^{5}$

This projects strengths lie in the targeted delivery of intervention to particular staff members. Identification of key professionals involved in the problem (junior doctors and nurses) provided a focus for each intervention. By altering the focus with each subsequent improvement cycle a different facet of the problem was addressed.

We identified several limitations of the project including the sample size which further work should look to address. A longer period of data collection is necessary to discern whether this strategy translates to a more sustained and robust improvement. Future work should be conducted in a different setting and with a larger sample size to assess generalisability and the statistical significance of any improvement seen.

Acknowledgements I would like to thank Dr Dan McKenzie, cardiology consultant, for supervising this project. His input was gratefully received when designing the proforma for Cycle3 and also in championing its use by the junior ward doctors.

I would also like to thank Jackee Phillips, the ward sister, for her energetic and ambitious involvement with the project. She provided a willing and helpful avenue of communication with the nursing staff as a body as well as preparing and overseeing the distribution of the visual reminders used in Cycle2.

Thank you both for your help and support.

Declaration of interests Nothing to declare

Ethical approval Ethical approval not sought, project deemed an improvement study with no study on human subjects.

Open Access This is an open-access article distributed under the terms of the Creative Commons Attribution Non-commercial License, which permits use, distribution, and reproduction in any medium, provided the original work is properly cited, the use is non commercial and is otherwise in compliance with the license. See:

- $\mathrm{http}: / /$ creativecommons.org/licenses/by-nc/2.0/

- $h$ ttp://creativecommons.org/licenses/by-nc/2.0/legalcode

\section{REFERENCES}

1. O'Driscoll BR, Howard LS, Davison AG, et al. BTS guideline for emergency oxygen use in adult patients. Thorax 2008;63:vi1-68 doi: 10.1136/thx.2008.102947

2. Austin MA, Wills KE, Blizzard L et al. Effect of high flow oxygen on mortality in chronic obstructive pulmonary disease patients in prehospital setting: randomised controlled trial. BMJ. 2010 Oct 18;341:c5462. doi: 10.1136/bmj.c5462

3. O'Driscoll R. British Thoracic Society Emergency oxygen audit report. BTS National Respiratory Audit Programme. Annual Report 2015.

4. Kumar P, Clarke ML. Kumar and Clark's Clinical Medicine 9th edition, Elsevier health sciences 29/06/16 Ch 24

5. Gatter M, Dixon G, Wall J, Mew E. Changing an ingrained culture: Improving the safety of oxygen therapy at University Hospitals Bristo NHS Foundation Trust. BMJ Quality Improvement Reports 2015; u203238.w1474 doi: 10.1136/bmjquality.u203238.w1474

6. NICE Clinical Guideline CG101. Chronic obstructive pulmonary disease in over 16s: diagnosis and management. Clinical guideline [CG101] Published date: June 2010 Accessed at https://www.nice. org.uk/guidance/CG101/chapter/1-Guidance\#management-ofexacerbations-of-copd 13/10/2016 